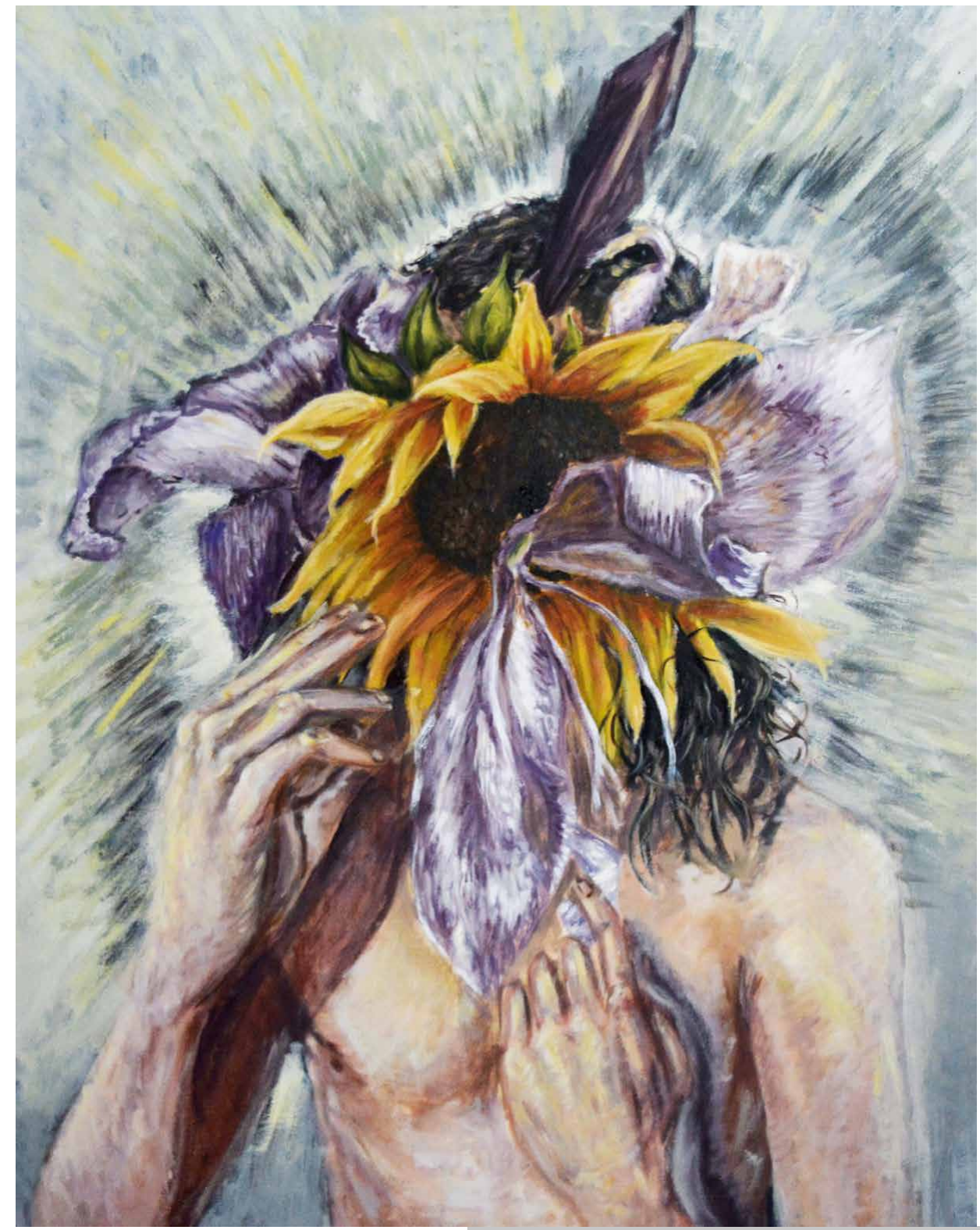

Artista invitado

Rafael Germán Rengifo Sánchez

Mi cabeza levita cuando se marchita

De la serie El jardín detrás de mi cabeza

Acrílico sobre lona

$76 \times 96 \mathrm{~cm}$

2020

Medellín 


\title{
La crisis de los sistemas democráticos en Nuestra América. Una reflexión psicopolítica a propósito de los estallidos sociales
}

\author{
Julián Darío Bonilla Montenegro (Colombia)* \\ Daniel Andrés Bonilla Montenegro (Colombia)**
}

\section{Resumen}

El actual sistema mundo ha presenciado diversos escenarios de confrontación desde los grupos subalternos que representan diversos modelos ideológicos frente a acciones generadas desde los centros de poder político estatal. Este artículo de reflexión busca analizar, desde una perspectiva psicopolítica, cuáles son los condicionamientos que se han impulsado, particularmente en Nuestra América, en las movilizaciones sociales contra diversas proyecciones políticas, independientes de su valoración ideológica. Se toma en cuenta el modelo del intuicionismo bergsoniano, recurriendo como criterio de análisis a los puntos convergentes que se han generado en los escenarios de protestas de la región. Se concluye que es necesario buscar alternativas para comprender los procesos de movilización y protesta social desde una perspectiva psicopolítica, así como la necesidad de reinterpretar los usos del poder en torno a estos fenómenos sociales en Nuestra América.

\section{Palabras clave}

Acción Colectiva; Movimientos Sociales; Protesta Social; Psicopolítica; Suramérica.

Fecha de recepción: julio de 2020 - Fecha de aprobación: febrero de 2021

\footnotetext{
* Abogado, Universidad Nacional de Colombia. Doctor en Ciudadanía y Derechos Humanos. Becario convenio 728/2015, Colciencias. Doctor (c) en Ciencias Políticas y de la Administración y Relaciones Internacionales. Docente investigador de la Universidad Libre de Colombia. Correo electrónico: juliand.bonillam@unilibre.edu.co - Orcid: 0000-0002-8059-1167 - Google Scholar: https://scholar. google.com/citations?user=OFPX_XUAAAAJ\&hl=es

** Psicólogo. Magíster en Desarrollo (c) Educativo y Social. Docente investigador de la Fundación Universitaria del Área Andina. Vinculado a Fundación Kadebra. Correo electrónico: dbonilla11@ areandina.edu.co - Orcid: 0000-0003-1549-6312
} 


\title{
Cómo citar este artículo
}

Bonilla Montenegro, Julián Darío y Bonilla Montenegro, Daniel Andrés. (2021). La crisis de los sistemas democráticos en Nuestra América. Una reflexión psicopolítica a propósito de los estallidos sociales. Estudios Políticos (Universidad de Antioquia), 61, pp. 205-229. DOI: 10.17533/udea.espo.n61a09

\section{The Crisis of Democratic Systems in Our America. A Psycho-Political Reflection on Social Outbreaks}

\begin{abstract}
The current world system has witnessed different confrontation scenarios from the subaltern groups, that represent various ideological models, facing the actions generated from the center of State political power. This reflection article tries to analyze, from a psycho-political approach, what are the conditioners that have propelled, particularly in the region of Our America, the social mobilization against diverse political range, independent of his ideological assessment. It takes into account the model of the Bergsonian intuitionism, resorting to the convergent points that have been generated in the scenes of protests in the region as a criterion of analysis. It concludes that it is necessary to look for alternatives in order to

[206] understand the processes of social mobilization and outburst from a psycho-political perspective, as well as the need to reinterpret the uses of power around these social phenomena in Our America.
\end{abstract}

\section{Keywords}

Collective Action; Social Movements; Social Protest; Psycho-Politics; South America. 


\section{Introducción}

En diversas regiones del mundo se ha presenciado un estallido social contra varias acciones políticas y sistemas de gobiernos. Desde las acciones de los chalecos amarillos en Francia hasta las protestas estudiantiles en Hong Kong, pasando por las fuertes manifestaciones en Iraq, todas han tenido como punto de inicio las protestas, valoradas por varios medios como políticas que buscan restringir derechos y prerrogativas a favor de la sociedad civil (Rachman, 2019, diciembre 29), abarcando procesos tanto en el ámbito político económico y social, pero que siguen siendo de difícil comprensión en términos comparativos debido a las disparidades que se presentan en los focos de protesta: regiones con altos niveles de Índice de Desarrollo Humano (IDH) como Francia o España frente a Estados en condición de fragilidad o debilidad institucional como Sudán o Venezuela.

Es importante considerar que «estos fenómenos sociales se producen en diferentes lugares, ya sean países del llamado sur global o "en vías de desarrollo", y/o [sic] del norte global o "desarrollados"» (Bonilla, D., 2020, p. 91), lo que ha decantado en que se puedan identificar brotes de movilizaciones sociales, protestas y manifestaciones frente a los modelos dominantes en diferentes latitudes.

De todos los procesos de movilización los que más llaman la atención en este artículo corresponden a Nuestra América: en Ecuador, Chile, Bolivia y Colombia se han presenciado, tanto con mayor y menor intensidad, como con mayor y menor consecución de ciertas metas, diversos contextos y formas de protestas, contra acciones políticas de diversa índole, tanto de carácter regional como de tipo nacional, que han tenido variaciones en su duración, así como impactos en la interacción de la sociedad civil con el Estado.

Venezuela puede ser incluido en este contexto de análisis, sin embargo, si se observa el espacio temporal de acción política y los factores que están anclados a favor y en contra de la figura de Nicolás Maduro y la trayectoria del proyecto político que actualmente él representa, surge una separación respecto a la súbita y, en cierta medida, sorprendente expansión de las protestas, las movilizaciones y acciones sociales en los cuatro Estados mencionados inicialmente.

En vista de lo anterior, este artículo busca configurar una reflexión respecto a las condiciones que se han presentado en Suramérica, un espacio 
geográfico claramente definible dentro de Nuestra América, donde se han presentado particularidades históricas propias que han hecho de estos espacios de protesta un ejercicio expresivo y simbólico del inconformismo social y la brecha social, política y económica que se presenta históricamente, lo que ha permitido brotes expansivos relacionados a la protesta social.

Para esto, el artículo se acerca a estrategias derivadas de la psicopolítica, referente conceptual del trabajo del filósofo coreano Byung-Chul Han (2014a) que identifica como técnicas de dominación elementos relacionados al desarrollo personal y al individual, tales como la motivación, la competencia, el desarrollo de proyectos, así como la posibilidad de generar iniciativas u optimizaciones, lo que lleva a repensar las formas de dominación que se han constituido en los últimos años por parte de diversos poderes opresores contra la sociedad civil, junto con las razones y las formas de legitimación que han tenido los grupos de movilización social en la región.

Sumado a esto, en este artículo se aborda la propuesta intuicionista de Henri Bergson, ya que se reconoce como una «multiplicidad cualitativa y virtual de unas direcciones diversas en las que se actualiza» (Deleuze, 1987, p.10). Por lo tanto, permite potenciar algunas características del pensamiento

[208 ] del autor coreano, puesto que enfatiza en el desarrollo de las percepciones y la relevancia de lo efímero para su constitución desde las tensiones que surgen entre lo efímero y la memoria, ya que es indiscutible que el territorio de la intuición real, es decir, instantánea, en la que se basa nuestra percepción del mundo exterior, es mínima en comparación con lo que agregamos a partir de nuestra memoria (Bergson, 2003); adicionalmente, al acercarse a lo político se identifica que se relacionan con la posibilidad de mediar y razonar, situación que implica mucho tiempo, yendo en contraposición a la experiencia a corto plazo (Han, 2020).

Por esta razón, resaltar el intuicionismo y la psicopolítica ayuda a destacar las implicaciones que tienen ciertas acciones en el plano de lo personal, social y político que son efervescentes, pero que no se mantienen en el tiempo, puesto que terminan siendo pasajeras, ubicando la discusión primeramente en las percepciones de los ciudadanos, un elemento fundamental en la compresión de este fenómeno social que entra en contraste con su permanencia o fugacidad, ya que en la percepción específica del sujeto intervienen tanto la memoria como la subjetividad. En este terreno, la subjetividad es producto de 
nuestra conciencia, que comienza con ser memoria, se extiende y se contrae en una intuición única de una pluralidad de momentos (Bergson, 2003).

Es más, las sociedades contemporáneas se caracterizan por tener una serie de respuestas que no permanecen en el tiempo, sino solamente se vuelven una mera expresión efímera de los intereses de las comunidades o sujetos. Considerar la indignación frente a muchos procesos en la actualidad nos acerca a reconocer a esta sociedad como una sociedad dominada y ruidosa, pero sin impacto sustancial, ya «La sociedad de la indignación es una sociedad del escándalo» (Han, 2014b, p. 21). Debido a que en la actualidad se da prioridad a lo instantáneo, desplazando elementos que han sido recurrentes en el desarrollo como sociedad y no se promueven cambios significativos, hay más conexión respecto con a momento efímero de efervescencia social que a una discusión y transformación en los diferentes ámbitos sociales.

Tener en cuenta la relevancia de los asuntos psicológicos y políticos de los seres humanos inmersos en las sociedades contemporáneas nos ayuda a discutir el interés por comprender los mecanismos de dominación que operan a partir de la psicopolítica, puesto que en la sociedad surge un «giro a la psique, y con ello a la psicopolítica, [...] relacionado con la forma de producción del capitalismo actual, puesto que este último está determinado por formas de producción inmateriales e incorpóreas» (Han, 2014a, p. 42). Esto nos alerta respecto a elementos críticos de la resistencia social al momento de considerar las formas en las cuales se han expresado las crisis democráticas en Nuestra América y el afán de los Estados por dominarlas, justamente, a partir de un condicionamiento que «permite intervenir en la psique y condicionarla a un nivel prerreflexivo» (p. 25).

Con respecto al ejercicio de construcción del diseño metodológico, este artículo toma como base el manejo de recolección de información a través de estrategias de minería de datos (Sedeño, 2015), ya que un gran núcleo de los criterios de identificación de estos procesos es propio del momento histórico inmediato (Arcudia y Pérez, 2014), pues se originan a través de la información digital que es posible recabar en Internet, pero previendo que surja información que pueda clasificarse como falsa o que sirva como dispositivo retórico (Gómez, 2016) que alteren la percepción sobre las motivaciones particulares que han impulsado el momento histórico de estos fenómenos sociales. 


\section{Caracterización del momento histórico en el sistema mundo}

Durante 2019 se vivió un momento histórico particular que permitió recordar otros escenarios que demostraban, en sus diversas etapas, la reivindicación de anhelos y pretensiones de los grupos subalternos en una clara oposición a la imposición de políticas dirigidas desde las altas esferas del poder político y que, claramente, se enfocaban en ampliar las brechas entre la población. Bajo este criterio, bien se podría pensar que 2019 fue un «año clásico para los disturbios populares» (Rachman, 2019, diciembre 29), con la característica de que, a diferencia de otros procesos, las regiones involucradas abarcaron una mayor cantidad de Estados, rompiendo la diferenciación clásica respecto a que el centro del sistema mundo protestaba bajo parámetros o condiciones diferentes a la periferia (véase cuadro 1).

Cuadro 1. Focos de protestas en el sistema mundo.

\begin{tabular}{|l|l|l|l|}
\hline \multicolumn{1}{|c|}{ Región } & \multicolumn{1}{|c|}{ País } & \multicolumn{1}{|c|}{ Inicio } & \multicolumn{1}{c|}{ Causa } \\
\hline $\begin{array}{l}\text { Oriente Medio } \\
\text { Ampliado' }\end{array}$ & Argelia & $\begin{array}{l}22 \text { de febrero de } \\
2019\end{array}$ & $\begin{array}{l}\text { Abdelaziz Buteflika anuncia su } \\
\text { aspiración a un quinto mandato } \\
\text { presidencial. }\end{array}$ \\
\cline { 2 - 4 } & Irak & $\begin{array}{l}1^{\text {o }} \text { de octubre de } \\
2019\end{array}$ & $\begin{array}{l}\text { Problemas económicos, posible } \\
\text { influencia iraní en el Gobierno. }\end{array}$ \\
\cline { 2 - 4 } & Líbano & $\begin{array}{l}17 \text { de octubre de } \\
2019\end{array}$ & $\begin{array}{l}\text { Creación de un impuesto para } \\
\text { aplicaciones telefónicas. }\end{array}$ \\
\cline { 2 - 4 } & Pakistán & $\begin{array}{l}27 \text { de octubre de } \\
2019\end{array}$ & $\begin{array}{l}\text { Denuncias de fraude electoral e } \\
\text { imposición de ayudas del Fondo } \\
\text { Monetario Internacional. }\end{array}$ \\
\cline { 2 - 4 } & Irán & $\begin{array}{l}15 \text { de noviembre } \\
\text { de } 2019\end{array}$ & Subida del precio de los combustibles. \\
\hline
\end{tabular}

\footnotetext{
${ }^{1}$ Oriente Medio Ampliado se refiere a la extensión de los países donde predomina el islam como religión, pero que no son árabes, como Irán, Afganistán o Pakistán, los cuales se encuentran en Asia, cubriendo la meseta iraní y la zona del Hindú Kush; esto en oposición a la categorización geopolítica convencional de Medio Oriente que restringe a los países de la península arábiga y, en ocasiones, del norte de África (Ozkan, 2011).
} 
La crisis de los sistemas democráticos en Nuestra América. Una reflexión psicopolítica...

Cuadro 1. Focos de protestas en el sistema mundo.

\begin{tabular}{|c|c|c|c|}
\hline Región & País & Inicio & Causa \\
\hline \multirow[t]{5}{*}{ Europa } & Francia & $\begin{array}{l}17 \text { de noviembre } \\
\text { de } 2018\end{array}$ & $\begin{array}{l}\text { Movimiento de los Gilets Jaunes contra } \\
\text { el alza de los combustibles. }\end{array}$ \\
\hline & España & $\begin{array}{l}14 \text { de octubre de } \\
2019\end{array}$ & $\begin{array}{l}\text { Sentencia del «procés» contra } \\
\text { independentistas catalanes. }\end{array}$ \\
\hline & $\begin{array}{l}\text { Reino } \\
\text { Unido }\end{array}$ & $\begin{array}{l}19 \text { de octubre de } \\
2019\end{array}$ & Manifestaciones contra el Brexit. ${ }^{3}$ \\
\hline & Georgia & Junio de 2020 & Reforma electoral. \\
\hline & Francia & $\begin{array}{l}5 \text { de diciembre de } \\
2019\end{array}$ & Reforma pensional. \\
\hline \multirow[t]{6}{*}{ América Latina } & Perú & $\begin{array}{l}30 \text { de septiembre } \\
\text { de } 2019\end{array}$ & $\begin{array}{l}\text { Crisis del gobierno del presidente } \\
\text { encargado, Ilamado a elecciones } \\
\text { anticipadas. }\end{array}$ \\
\hline & Ecuador & $\begin{array}{l}1^{\circ} \text { de octubre de } \\
2019\end{array}$ & $\begin{array}{l}\text { Acuerdo con el Fondo Monetario } \\
\text { Internacional, se retira el subsidio a los } \\
\text { combustibles. }\end{array}$ \\
\hline & Chile & $\begin{array}{l}19 \text { de octubre de } \\
2019\end{array}$ & $\begin{array}{l}\text { Aumento del pasaje del metro de } \\
\text { Santiago. }\end{array}$ \\
\hline & Bolivia & $\begin{array}{l}21 \text { de octubre de } \\
2019\end{array}$ & $\begin{array}{l}\text { Denuncias de fraude en las elecciones } \\
\text { presidenciales. }\end{array}$ \\
\hline & Panamá & $\begin{array}{l}30 \text { de octubre de } \\
2019\end{array}$ & Reforma constitucional, oposición social. \\
\hline & Colombia & $\begin{array}{l}21 \text { de noviembre } \\
\text { de } 2019\end{array}$ & $\begin{array}{l}\text { Cambios en la política social y } \\
\text { económica, corrupción y desigualdad. }\end{array}$ \\
\hline Asia & Hong Kong & $\begin{array}{l}15 \text { de marzo de } \\
2019\end{array}$ & $\begin{array}{l}\text { Reforma legal para permitir la } \\
\text { extradición de ciudadanos hongkoneses } \\
\text { a China. }\end{array}$ \\
\hline
\end{tabular}

Fuente: elaboración propia a partir de Pasquali (2019, noviembre 21); BBC News Mundo (2019, noviembre 14; 2020, enero 31); Saura (2019, diciembre 1.9); Rachman (2019, diciembre 2019); El Comercio (2020, enero 9).

\footnotetext{
${ }^{2}$ Entiéndase que corresponde al Reino Unido de la Gran Bretaña e Irlanda del Norte. Las movilizaciones fueron más fuertes en Inglaterra y Escocia, dos de los cuatro países que conforman en Reino Unido (Ramos, 2019, octubre 19).

${ }^{3}$ Expresión que combina las palabras exit y british. Surgió debido al triunfo del referendo que permitió el retiro del Reino Unido de la Unión Europea y que se celebró en junio de 2016 (BBC, 2020, enero, 31).
} 
Con el cuadro 1 se puede rescatar para este ejercicio, en primer lugar, que los procesos de movilización y acción social han tenido distintas motivaciones, que están articuladas a diferentes procesos, por ejemplo, políticos — fraude electoral o reelección—, económicos — subida de precios de transporte público, modificación en sistemas de seguridad social- e incluso legales - transformación de los modelos de punición-; en segundo lugar, llama la atención la proximidad temporal en la aparición de los procesos de protesta, lo que haría suponer que esto correspondería a una suerte de efecto dominó, en donde se considera que si algo pasa en una región debe ocurrir también en otra.

Es necesario enfatizar el hecho de que los procesos de movilización pudieron mantener un contacto de información permanente debido a la difusión masiva a través de redes sociales. Este aspecto es significativo, ya que la adquisición y repercusión del uso de las nuevas tecnologías de información y comunicación sirven para transformar los criterios respecto a por qué se genera la movilización, cuáles son sus causas y cuáles podrían ser sus consecuencias (Resina, 2010), algo que tuvo su primer momento de identificación como factor de influencia en los procesos del sistema internacional, a consecuencia de las acciones presentadas en el contexto de los levantamientos sociales en la Europa que vivió las afectaciones de la crisis económica de 2008 y en donde se comenzó a visibilizar el impacto de los procesos del manejo de redes sociales (Bonilla, J., 2013), fenómeno etiquetado como «efecto Facebook», demostrando que «las tecnologías y la comunicación jugaron sic] un papel clave en las luchas por una democratización [...] en un movimiento de abajo arriba» (Marí y Santos, 2016, p. 3). ${ }^{4}$

\section{El contexto nuestroamericano}

Ahora bien, Nuestra América también fue una región que presenció diversos espacios de movilización social como forma de oposición a varias de las acciones presentadas desde los gobiernos, tanto de forma explícita

\footnotetext{
${ }^{4}$ Son necesarias dos caras para comprender este fenómeno: por un lado, la visión positiva, incluso algo idealista, ha querido ofrecer la idea de que el uso de las redes sociales ha permitido una ruptura con los modelos de control tradicionales, así como la posibilidad de generar espacios de difusión que no tienen controles de censura por parte de los gobiernos. Sin embargo, en los últimos años, con el auge del manejo de las noticias falsas - fake news - se ha podido entender que esto ha sido una estrategia muy bien desarrollada desde sectores autoritarios para mantener una situación de permanente temor a la sociedad civil, fortaleciendo sus posturas conservadoras o retardatarias, así como aumentando sus posibilidades de triunfo electoral.
} 
como implícita. Es importante mencionar que este tipo de movilizaciones no tuvieron un foco ideológico común, ya que se vieron involucrados en este escenario gobiernos tanto de izquierda - Bolivia - como de derecha —Ecuador, Chile y Colombia-; además, como es posible identificar en el cuadro 1, fueron condiciones diversas las que llevaron a que se presentaran las acciones de manifestación y movilización social, aunque una excepción a este proceso corresponde al caso colombiano, ya que esta se podría considerar más coordinada en comparación con las demás, debido a que su surgimiento fue acordado por diversos sectores sociales sobre la base de una alta acumulación de descontento social, en tanto que los otros países presenciaron acontecimientos concretos como la eliminación de subsidios o resultados electorales.

El mapa 1 puede permitir un acercamiento más detallado en relación con los espacios geográficos en donde se efectuaron los procesos de movilización y sus causas.

Mapa 1. Protestas y movilizaciones en Nuestra América.

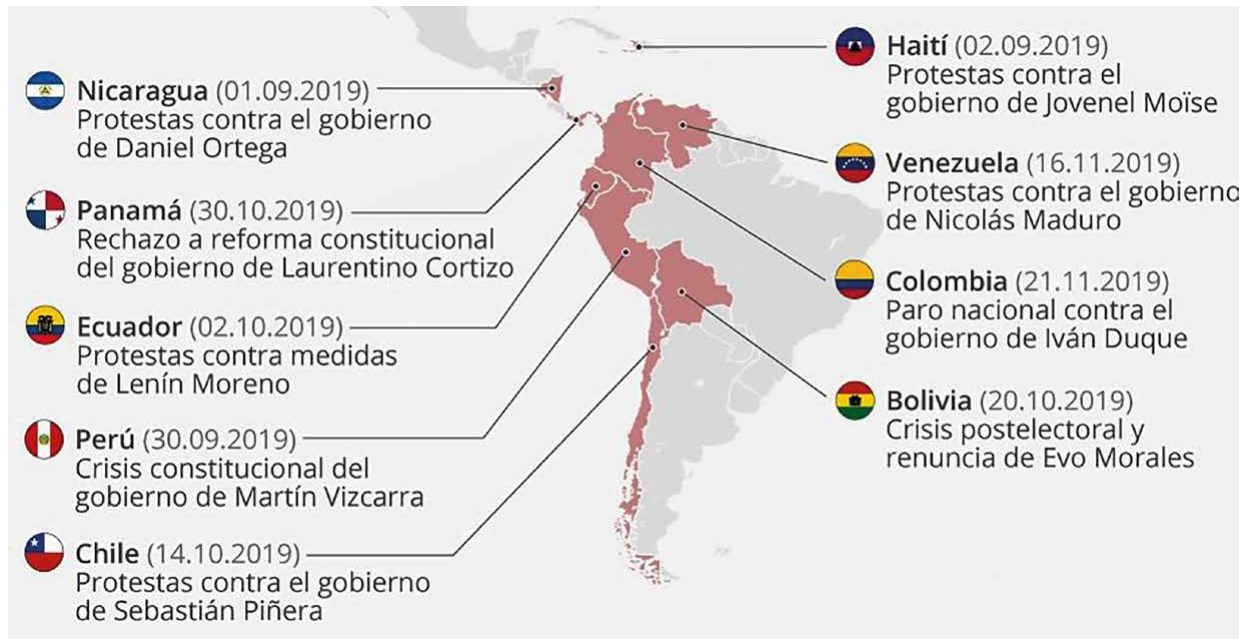

Fuente: Pasquali (2019, noviembre 21).

Como se puede apreciar en el mapa 1, fueron diversos los criterios que permitieron que se generara un proceso de activismo social muy marcado en la región, teniendo diferentes grados de acción social y de resultados alrededor de los motivos de las protestas. Esta diversidad se convierte en un tema de interés para su investigación y conceptualización. Por esta razón, 
es importante sedimentar algunos elementos que puedan ser una ruta de análisis para este fenómeno social. Lo que se propone a continuación corresponde a la caracterización teórica que sirve para poder comenzar con el reconocimiento de ciertos aspectos comunes vinculados con la connotación de una psicopolítica que se vincula a los factores de movilización.

\section{La psicopolítica y formas de transición de la conducta política}

En este ejercicio académico se busca hacer la construcción de un análisis tomando como criterio la base conceptual de la propuesta de Han (2014a), la cual considera que se han configurado nuevas estrategias de poder desde las formas políticas neoliberales, construyendo procesos de control «inteligente» en donde se ven involucrados los procesos y las prácticas actuales de difusión de la información por parte de los medios de comunicación de masas, así como la cada vez más elevada dependencia a las redes sociales.

De acuerdo con el autor, existe una serie de patrones de comportamiento que afectan el inconsciente colectivo — consideración relevante dentro de los ejercicios de estudio de la psicología política (Ernst, 2010)— mediante diversas

[214 ] formas de manejo y regulación de las emociones, con el fin de enfocarse en procesos de consumo que impidan que realmente se desarrollen procesos tipo revolucionario. Este ejercicio conlleva a reconocer que determinados patrones de conducta pueden servir para generar procesos de modificación o transformación en diversas formas de representación — cómo entiendo- e identificación — quién soy— de tipo social, lo que puede conducir a que se configuren condiciones específicas de acción que impulsen a los individuos a actuar de acuerdo con criterios subjetivos que pueden verse identificados con patrones de tipo colectivo, los cuales mantienen la actual hiperindividualización que permite que sea el mismo individuo quien manifieste, creyendo que actúa de forma autónoma, sus patrones de conducta, los cuales, en ocasiones, considera que deben ser reflejados mediante espacios de comunicación digital como las redes sociales.

Lo expuesto sirve para poder reconocer que se han producido en la región escenarios de movilización que pueden basarse en una serie de criterios compartidos, pero que, en esencia, son formas subjetivas que se enmarcan en las acciones o prácticas psicosociales desde donde pueden tener una capacidad vinculante o efectiva, en relación con los resultados de 
la acción social para efectuar procesos de transformación en las formas de interacción de todo el entramado social. Esto genera un terreno peligroso al reconocer que las tecnologías y lo entendido como mass media permiten que el sujeto se aísle significativamente y pierda su posibilidad de agenciamiento respecto a las dinámicas propias de la protesta y movilización social. En este punto cabe resaltar que las diferentes estrategias desde la psicopolítica se expresan al momento que un sujeto, recurriendo a diferentes redes sociales o tecnologías, se manifiesta en la virtualidad, desajustando las posibilidades de generar procesos de cambio social.

En suma, es un peligro latente frente a las diferentes estrategias del Estado para mantener el control y reducir especialmente las condiciones del ser humano a una fracción que aparenta contacto pero que carece de este. En este sentido, cabe realizar la siguiente pregunta: ¿cómo reconocer si los procesos políticos tienen connotaciones derivadas de las formas de interacción de tipo psicológico? Un valor de apreciación proviene de los aportes establecidos desde el campo de la psicología política crítica, una apuesta que considera que: «En la medida en que una actividad promueva los intereses de un determinado grupo social y que afecte o influya en el equilibrio de las fuerzas sociales y en el orden social tal como se encuentran en un determinado momento, esa actividad tiene carácter político» (Bustos, 1990, p. 214).

Al momento de conformar una analogía en relación con los criterios de reflexión para los procesos de las manifestaciones sociales que se han presentado en la región, se puede identificar claramente que existe una razón y motivación política que han generado el impulso a esta clase de actividades; sin embargo, lo que hace falta por identificar es el reconocimiento, en caso de que exista, de los puntos en común que se pueden presentar en estos procesos.

El análisis respecto de estos procesos de interpretación se aplicará el proceso intuicionista de Bergson (2003). Esta propuesta sirve para explicar «la relación del yo con el universo que le rodea desde el ámbito de la subjetividad [...] que será uno de los elementos decisivos para dar respuestas a las múltiples dicotomías del mundo moderno» (Mascato, 2009, p. 67). Esto, teniendo en cuenta que el Estado condiciona a partir del uso de las tecnologías, expulsando a las personas de las dinámicas sociales y buscando estrategias para responsabilizarlos de sus acciones sin posibilidad de confrontar 
las inconsistencias del Estado, lo que resulta en un proceso de subjetivación frente a las dinámicas que les imponen a los sujetos.

Conforme a lo anterior, Han (2014a) afirma que «Hoy se realiza otro cambio de paradigma. El panóptico digital no es ninguna sociedad biopolítica disciplinaria, sino una sociedad psicopolítica de la transparencia» (p. 106). Con esta expresión el autor define una sociedad que se embarca en el libre acceso a la información, en la cercanía, en lo uniforme, elementos que en apariencia distinguen la libertad y la democracia, pero que distinguen el control y la dominación para evitar inconformidades entre los ciudadanos. De hecho, «la transparencia es en sí positiva. No mora en ella aquella negatividad que pudiera cuestionar de manera radical el sistema económico-político que está dado» (Han, 2013, p. 22). Además de esto cabe señar que «la violencia de la positividad no presupone ninguna enemistad. Se despliega precisamente en una sociedad permisiva y pacífica. Debido a ello, es menos visible que la violencia viral» (Han, 2012, p. 22). Esto demanda repensar estrategias de comprensión, emancipación y transformación que aludan a la reflexión crítica de los cambios y convulsiones propios de las estrategias de dominación de las sociedades.

\section{[216] 4. Nuestra América: crisis democrática y procesos de transformación}

La primera forma de reconocimiento común de los procesos de movilización corresponde al hecho que estos fueron iniciados y sostenidos en su mayoría por una nueva clase social: el precariado (Standing, 2013). Este corresponde a un proceso variado dentro de las categorías económicas y sociales vinculantes, pero que se caracteriza por la ausencia de una identificación plena como clase colectiva. Por regla general, las condiciones de precarización se identifican como una tendencia dominante que establece patrones concretos de comportamiento sobre este grupo, bajo el temor de perder varios de lo que se consideran son beneficios adquiridos, pero que, en la realidad, retomando el ejercicio de análisis de Han (2014a), solamente corresponde a bienes de consumo descartables que cada vez se consideran con una mayor fortaleza y dependencia para su interacción con los individuos.

Otra característica vinculante de todos los procesos de movilización que se han presentado en la región corresponde al hecho de la constitución de un criterio de abandono por parte de la clase gobernante, considerando que muchas de las leyes o reglamentaciones son una burla para este grupo social 
diverso (Bartolomé, 2012). Ahora, existe una aparente armonización entre las clases sociales de la base de la pirámide socioeconómica. La concentración de la riqueza se acentúa cada vez más y esto conlleva a que se vea afectada la representación política de estos grupos ante las instancias de deliberación democrática, lo que se demuestra en el hecho de que se «recrean y reinventan un modo específico de ...] rebelión [...] que en su masividad y espontaneidad arrasa con la escasa legitimidad política» (Grüner, citado por Torres y Arúe, 2013, p. 113).

Y no solo esto, se alerta respecto a la pérdida de poder transformativo de estos movimientos sociales que surgen de la ruptura del pacto social, ya que terminan siendo una respuesta pasajera, pero sin injerencia política que logre modificar o transitar hacia un cambio: «no se forma ninguna comunidad en sentido enfático. Surgen solamente acumulaciones o pluralidades casuales de individuos aislados para sí, de egos, que persiguen un interés común, o se agrupan en torno a una marca (Brand communities: comunidades de marca)» (Han, 2013, p. 93). Lo que alerta respecto a la poca posibilidad de cambio dentro de la sociedad y a estrategias que surgen dentro de los diferentes bloques del poder para afincarse en el lugar privilegiado de la desigualdad social, excluyendo al resto.

Sumado a esto, las élites, las cuales históricamente han mantenido el control del poder político en estos países - con excepción del caso boliviano, en donde se podría configurar que el sujeto reprimido es la misma élite racista que considera que se ha presentado una pérdida de valores y principios correctos con la presidencia de Evo Morales y su reivindicación de un proyecto desde la base indígena-, también representan los sectores más aventajados social, económica y educativamente, lo que conlleva a que consideren que deben permanecer aislados del resto del cuerpo social (Alvarado, Ospina y García, 2012).

Incluso al lograr construir procesos simbólicos de interacción, que también pueden ser hábilmente manejados por medio de las redes sociales, lo que finalmente se consigue es una fragmentación de la idea de nación, entendida aquí como un criterio de unificación social de carácter cultural, así como el aumento de la desconfianza en la credibilidad de los procesos democráticas de participación electoral, pues, ¿̇e qué sirve elegir mediante el voto si al final se generan procesos de opresión y menosprecia a la base electoralmente activa? 
Christopher Lasch (1996) reconoce que desde las mismas élites se genera ese proceso de fragmentación del pacto social, de aquella idea democrática que supone que los procesos de organización del cuerpo político y social dentro del Estado corresponden a un contrato social, construyéndose un modelo narcisista de democracia en donde se traza «una estructura compartida que filtra el modo que tenemos de razonar, o más bien, de opinar» (Martínez, 2015, p. 191), lo que implica que se reconfiguran nuevas formas de diferenciación en los campos de interacción social, político, económico y cultural, instituyendo de esta manera formas de reducción de la calidad de la democracia, la cual termina siendo referenciada en el campo discursivo como el dispositivo formal, legal y periódico por medio del cual se efectúa la transición de determinados gobernantes, pero que, en gran medida, lo que establece es el aprovechamiento del Estado por parte de las clases gobernantes mediante transiciones entre diversos grupos en la cúspide de la pirámide de los espacios de gobierno estatal.

\section{La oposición, la multitud y la movilización}

Cuando Michael Hardt y Toni Negri (2004) quisieron ofrecer una idea acerca de la multitud y su capacidad de ser un movimiento de transformación

[218] «que armonizaba con las fuerzas y los deseos que se extendían horizontalmente por todo el globo, redoblados por el descubrimiento de otras relaciones y otros territorios» (Hardt y Negri, 2002, p. 83), creyeron encontrar un referente particular que serviría como punto de organización de la acción social que lograra enfrentar las acciones sistemáticas de imposición de un sistema de dominación económico inspirado en los criterios del orden neoliberal. Esto podía configurar condiciones de manifestación variadas de acuerdo con los puntos de descontento que fueran reconocibles desde el proceso mismo de la interacción social. Pero «para que ese descontento pueda traducirse en acción política, es necesario que responsabilicemos a algún agente externo de esa situación. Si no existe ese proceso de atribución externa, ya sea al gobierno a otro grupo social, la movilización no tiene sentido» (Sabucedo, Rodríguez y López, 2000, p. 347. Énfasis añadido).

Este criterio de atribución se ha construido dentro del escenario nuestroamericano tomando como base la asignación de justificantes para la movilización que, siendo particularmente subjetivos, no han logrado aglutinarse bajo un liderazgo específico, lo que conlleva a que puedan presentarse diversas formas de interpretación sobre los planes de acción 
y las estrategias que deben efectuarse durante y después del proceso de movilización, una vez se hayan evaluado sus resultados. No obstante, también la movilización reciente ha demostrado que se puede construir un proceso acéfalo en cuanto se reconoce que las formas de divulgación, así como las propuestas de reorganización de los diversos aparatos estatales, se construyen mediante formas de interpretación totalmente aisladas de la realidad social, en donde prevalece la identificación de una estética (Fernández, 2003) que representa el estilo social construido desde el sector de las élites y que configura, a modo de cascada, la normalización de los demás procesos de interacción en las demás capas sociales.

Adicionalmente, existe otro criterio compartido que ha tenido eco en las experiencias enmarcadas en los procesos que se comienzan a gestionar en la etapa posterior al nacimiento de la protesta. En el cuadro 1 se comentan las razones por las cuales se considera que se inician los movimientos de protesta en los países, sin embargo, es necesario enfatizar que estos no han sido los únicos criterios de legitimación social de la movilización o protesta social. Es posible considerar que en varios casos, siendo el más simbólico Chile, las protestas no se han detenido ${ }^{5}$ únicamente porque desde Presidencia se haya revocado la medida que buscaba aumentar el costo del pasaje en el metro de Santiago, sino que el proceso fue extendiéndose para llegar incluso a exigir la destitución del presidente, un cambio real en el sistema de pensiones o la realización de una Asamblea Constituyente para la modificación de la Constitución Política, herencia del régimen militar de Augusto Pinochet. Este espontaneísmo ha servido para lograr articular todas las situaciones de malestar social (Moriconi, 2009) que ha vivido gran parte de la sociedad chilena.

Es desde el ejercicio de la movilización y su espontaneidad que se marcan algunas inquietudes respecto a la representatividad que han tenido los procesos de acción social. En primer lugar, se configura cierta ambigüedad sobre cuál puede ser el impacto real de todas estas medidas y pretensiones generadas sin una base justificante clara, lo que puede permitir que se presenten conflictos de interpretación entre los diferentes grupos que han conformado la base de la movilización, ya que es probable que cada sector comience a interpretar las condiciones de éxito o fracaso de acuerdo con

\footnotetext{
${ }^{5} \mathrm{Al}$ momento de redactar este artículo, incluso con la vigencia de la pandemia del SARS-CoV-2, la cual permitió la conformación de nuevos modelos de represión estatal como medida para contener la expansión de contagios.
} 
sus propias valoraciones, considerándolas más importantes que las demás. En segundo lugar, al momento de identificar las circunstancias del surgimiento de la espontaneidad es probable que se construyan desde los centros de poder mecanismos adicionales de regulación y adaptación que permitan mantener vigente la idea, incluso cuando se sostenga el statu quo (Garzón, 2008).

En una comparación de tipo diacrónico es posible reconocer que esto es parte del legado del proceso anclado al Mayo del 68, en el cual, luego del estallido de la movilización de los jóvenes estudiantes franceses —que como es posible constatarlo en la coyuntura actual, generó una resonancia muy alta que permitió su expansión por otras regiones del mundo-, las élites en el poder reorganizaron parte de sus discurso, tomaron para sí algunas de las categorías y conceptos construidos desde la movilización, ajustaron su campo definicional y lograron mantener una suerte de expectativa sobre la posibilidad real de lograr un cambio.

De forma similar se puede pensar en lo que ocurrió luego de la crisis de las hipotecas sub prime en Estados Unidos, cuando se creyó que incluso el sistema capitalista entraba en una fase de crisis inevitable; o durante 2010 y 2011, cuando se generó el proceso de transformación social en los países [220] árabes, que llevaron al derrocamiento de algunos gobiernos. El sistema capitalista sigue vigente, cada vez con mayores ambiciones de expansión y de reorganización para satisfacer la acumulación de capital y, en el Medio Oriente, varios Estados entraron en guerra civil o se han instaurado gobiernos militares o de otras formas de autoritarismo - democracias iliberales o liderazgos autoritarios-.

Este punto es importante observarlo dentro del contexto colombiano, fenómeno que se pensaba inédito en la historia del país - Colombia se ha caracterizado por la poca movilización social, tal vez como consecuencia de las diversas formas de violencia armada en su historia y que han reprimido la posibilidad de su aceptación por el temor generado-, contando como excepcional el Paro Cívico Nacional de 1977 (Archila, 2016). Esto permitió que se haya despertado en diversas capas sociales las posibilidades de lograr cambios particulares a partir de las diversas críticas promovidas en este momento de acción social. Sin embargo, esto no ha logrado tener la fuerza suficiente para impedir que el gobierno siga insistiendo en las reformas económicas de corte neoliberal, ya que se mantiene la idea de generar 
reformas que mejoren algunos indicadores económicos, pero que también lograrán aumentar las brechas de desigualdad en el país.

Esto no es así en los casos de Ecuador o Bolivia, en donde la acción social, especialmente desde la base indígena, siempre ha desempeñado un campo de identidad y representación política muy fuerte que ha logrado demostrar la capacidad del pueblo de estos Estados cuando busca garantizar el cumplimiento de sus exigencias, independientemente de las formas de gobierno o de las interacciones, a veces violentas, que pueden presentarse frente a la fuerza pública.

\section{6. ¿Por qué se habla de crisis? ¿Hay alternativas de solución?}

En todo el proceso de presentación de las características de los procesos de movilización social en la zona sur de Nuestra América se puede identificar la existencia de una crisis democrática, la cual no puede identificarse bajo un criterio convencional, esto es, existencia reiterada de fraudes electorales o de acciones que impiden un verdadero ejercicio de actividad democrática en la región, sino por el hecho de que se ha perdido la confianza en lo que resulta posterior a la actividad electoral. Hay quienes consideran que, en ese caso, siempre ha existido crisis de la democracia porque siempre ha habido diversas formas de fraude. Lo que debe considerarse es que, a pesar de las acciones de corrupción electoral presentes en todo proceso de participación política electoral, no se puede considerar la presencia de escenarios masivos que impidan la revisión y supervisión de los resultados, así como la acumulación excesiva de votos en procesos de carácter nacional a favor de un determinado candidato. En la actualidad, la sociedad vive en un constante proceso de mediatización y conformación de identidades políticas, las cuales buscan ser cooptadas por parte de determinados grupos dentro de las clases políticas con el fin de garantizar la legitimación de su accionar, ofreciendo la imagen de un contacto directo, así como del reconocimiento de que ese grupo social realmente se encuentra afectado y, por lo tanto, realmente se trabajará para otorgarle su ayuda. Sin embargo, la clase política está cada vez más alejada de la ciudadanía y no tienen la capacidad real de comprender su entorno interactivo, no logran demostrar ni ofrecer alternativas que satisfagan lo suficiente a la población. 
En este caso, se puede hablar de crisis porque existe un malestar difuso que representa «una sobreinterpretación del estado de ánimo de la población y de las consecuencias que se derivarían de este» (Beyer, 2015, p. 109), lo que altera la confianza que la sociedad debería en el Estado. La crisis, entonces, debe considerarse democrática porque quebranta la necesidad de construir una representación ciudadana y una adecuada interacción entre el grupo de gobernantes frente a los gobernados, porque aquellos generan una «incapacidad de entregarles certeza a los ciudadanos en áreas que son claves para su seguridad económica y personal» (p. 109).

¿Existen algunas formas de solución?, ¿son viables? Se puede caer en un error de interpretación si se considera que este trabajo es un ejercicio de carácter prospectivo. Lo que se busca es identificar algunos puntos sobre los derroteros de la movilización, estableciendo qué condiciones sirven para reforzar su legitimación y que esto genere un reconocimiento serio desde las élites gobernantes, evitando que sea desde esas mismas élites que se configuren procesos de difusión de información falsa en contra de los valores de la movilización y la acción social.

En primer lugar, debido a la gran cantidad de información que [222] diariamente se recibe mediante diversas formas de comunicación en red, se han desintegrado en gran medida los criterios de validez de muchas de las propuestas de necesidad de reforma o se ha radicalizado la respuesta frente a estas, lo que ha llevado a considerar elementos como las fake news o la posverdad, los cuales deberían entrar en el debate público para valorar su efecto y sus usos en relación con el manejo emocional y la conformación de las decisiones políticas.

En Bolivia, por ejemplo, luego de la renuncia de Evo Morales, comenzó un proceso de aproximación política por parte de grupos fundamentalistas evangélicos que consideran que debe ser más importante la biblia que la Constitución o que el único criterio para reorganizar el país es la palabra de Dios (Expansión, 2019, noviembre 15). En Colombia, como una forma de oposición a las actividades del Paro Nacional se ha difundido la idea de la infiltración de la guerrilla en las marchas, la presencia de «vándalos» o incluso la desproporción de considerar que todo el proceso de movilización es una estrategia coordinada por parte de actores externos (Jojoa, 2019, noviembre 17). Situación similar a la que se vivió en Ecuador (Infobae, 2019, octubre 10) y Chile (Sfeir, 2020, junio 7) donde se culpaba a actores extranjeros de ser los 
determinadores de las protestas como parte de una estrategia para tomarse el poder. Si no se logra un modelo de revisión y filtro de la información este tipo de acciones podrán seguir siendo efectivas y afectarán cualquier intento adicional de buscar reformas a las problemáticas sociales de la población.

De igual forma, se hace necesario construir procesos en donde el sujeto tenga una verdadera capacidad de participación que vaya más allá de su vinculación a los procesos de movilización. Es necesario una valoración más precisa del qué se quiere y cómo se puede llevar a cabo, que quiebre la identificación con simples vaguedades que no logran aglutinar realmente más sectores sociales. No es posible, bajo este criterio, excluir al observador ni la construcción de significados que se generen gracias a las acciones de protesta, pretendiendo aislarlas del entorno social que han permitido su surgimiento (Arcila, Mendoza, Jaramillo y Cañón, 2010).

Es importante, además, que se logre una ruptura con el modelo que se ha venido implantando para justificar el desarrollo del Estado. Esa visión estructural que suele ofrecerse bajo la condición de que lo propuesto es un verdadero modelo de éxito desconoce las disparidades que se presentan entre las capas sociales (Beyer, 2015), y solamente funciona como una suerte de anteojeras para los diseñadores de políticas públicas que consideran que sus modelos no aceptan ninguna forma de cuestionamiento desde la ciudadanía porque, al final de cuentas, toda la ciudadanía recibirá, en mayor o menor medida, beneficios por parte de estas políticas.

\section{A modo de conclusión}

Las democracias han fallado desde su propuesta inicial y se ha visto cómo estas se transforman en formas de gobierno que cooptan el poder para satisfacer determinados beneficios a ciertos sectores de la sociedad. Así, lo que realmente se presenta en el momento histórico actual, más que un sistema democrático es una forma de oligarquía electiva (Watson, 2005), lo que permite que se generen las condiciones de insatisfacción que hacen que se viva bajo un proceso de crisis de democracia manifestado en los últimos meses en las diversas formas de movilización social en algunos países de la región.

Por otro lado, la responsabilidad de los sujetos debe ser resignificada, reubicada frente a las posibilidades de cambio y las relaciones que se tejen 
entre la ciudadanía, ya que las sociedades contemporáneas se han encargado de individualizar y promover la autoexplotación de los sujetos, puesto que en la actualidad «quien fracasa en la sociedad neoliberal del rendimiento se hace a sí mismo responsable y se avergüenza, en lugar de poner en duda a la sociedad o al sistema» (Han, 2014a, p. 18). Esto representa, en cierta medida, un contrapeso para los movimientos, resistencias, manifestaciones y protestas, dispersando la posibilidad de transformación en el contexto. Ya que el diseño de las sociedades contemporáneas, desde la mirada de Occidente, acoge la necesidad de promover el autoaislamiento y disminuir los vínculos sociales de las personas, e incluso se ha comenzado a desvalorizar la agremiación o los procesos comunales porque el modelo no permite una vinculación potenciable.

Asimismo, se debe procurar ajustar los procesos colectivos para que no terminen siendo dispersados por las estrategias de dominación de quienes ejercen el control, puesto que se han dedicado a generar procesos en los cuales se deslegitima, invisibiliza y dispersan las apuestas de resistencia, de hecho, «las olas de indignación muestran una escasa identificación con la comunidad. De este modo, no constituyen ningún nosotros estable que muestre una estructura del cuidado conjunto de la sociedad» (Han, 2014b, p. 13). Por lo cual, posibilitar aspectos relacionales, personales y colectivos que fortalezcan las prácticas de cuidado y autocuidado de la ciudadanía puede contribuir a fortalecer los grupos y las comunidades que pretenden resistir en las sociedades contemporáneas.

En suma, es importante reconocer espacios de acción social como las vocerías y los liderazgos, puesto que son reconocibles dentro de las dinámicas sociales, colectivas y comunitarias que vinculan la ciudadanía con el Estado; así como revisar los procesos, estrategias y tácticas que se vinculan al ejercicio del poder al momento de acceder a un espacio político determinado, ya que los liderazgos políticos actuales se han encargado de desacreditar los procesos democráticos que los han llevado al poder, permitiendo de forma directa o indirecta el debilitamiento de las instituciones estatales que no les sean funcionales a sus intereses (Levitsky y Ziblatt, 2018). Por ese motivo una de las pocas instituciones que no se ven afectadas son la militar y de policía, ya que deben seguir encargándose de la seguridad de determinados espacios geográficos y sectores sociales. Este proceso de debilitamiento ha sido una de las condiciones por las cuales se puede apreciar el descontento y el desánimo 
de una amplia capa de grupos sociales en relación con sus perspectivas hacia el futuro o en la inserción a procesos de cambio.

Las diferentes estrategias que se han llevado a cabo por parte de los países han logrado impulsar una suerte de dictadura invisible que desarticula las posibilidades de movilización social, un impulso que tiene el riesgo de perderse en la innumerable producción de información y en la hipervelocidad en la que nos encontramos inmersos. En este sentido, se debe reconocer la propuesta de la psicopolítica respecto a la manera en que se han individualizado y responsabilizado a los sujetos durante el desarrollo de nuestras sociedades basados en la autoexplotación y en la positividad.

Lo que debe tenerse en cuenta corresponde al hecho de replantear las formas de movilización social, logrando identificar los criterios comunes y acciones sociales entre los distintos sectores que han logrado su participación, aunque ahora es más que todo una práctica subjetiva, tratando de alejarse de las formas de control que se han gestado mediante las estrategias de dominación que ha logrado identificar Han (2014a), las cuales priorizan elementos psicológicos y que atacan al sujeto enclaustrado en su individualidad. Por otro lado, el control psicopolítico que han querido construir las élites debe ser afrontado mediante otras formas y estrategias de acción que permitan resistir a la individualización, a la autoexplotación, a la aparente libertad que nos han dado dentro de la sociedad, así como a evitar la recepción permanente de información falsa y el silenciamiento sistemático de la resistencia social.

Asimismo, es importante operar en los diferentes niveles de análisis y comprensión respecto a las acciones y comportamientos de las manifestaciones, protestas o movilizaciones, ya que a partir de los vínculos que se construyen en nuestras sociedades es posible reconocer otras formas de expresarlas, lo que lleva a buscar estrategias alternativas para acercarse a dichos fenómenos sociales, los cuales se consolidan en diferentes lugares y latitudes, tensionando la relación entre ciudadanía y Estado.

Los procesos de manifestación no han acabado en la región, algunos han perdido su impulso, otros han mutado para poder vincularse a otras formas de acción social. Lo que debe tomarse en consideración es la necesidad de que la base social se mantenga activa y logre servir de contrapeso real a las acciones de debilitamiento de la democracia que se 
han desarrollado en la región, conformando de esta manera alternativas reales y eficientes de comunicación y reconocimiento frente al Estado. Esto no puede considerarse como una derrota, sino un llamado de atención para agenciar estrategias de transformación y validar la legitimidad de las protestas y los movimientos sociales.

Esto permite enlazar procesos alternativos que buscan una transformación en Nuestra América, pero que están en riesgo de sufrir alteraciones a partir de los nuevos modos de ejercer el poder e incluso deslegitimar sus acciones en los ámbitos interno y externo del Estado, por lo cual es importante establecer criterios y puntos de fuga para el reconocimiento de alternativas de acciones que puedan vincularse a los estallidos sociales dentro de los territorios, logrando una reinterpretación de estas acciones en diferentes ámbitos, contextos y escenarios de participación, y no meramente una respuesta masificada efervescente, pero sin contundencia.

\section{Referencias bibliográficas}

1. Alvarado, Sara; Ospina-Alvarado, María y García, Claudia. (2012). La subjetividad política y la socialización política desde las márgenes de la psicología

[226] política. Revista Latinoamericana de Ciencias Sociales, Niñez y Juventud, 10 (1), pp. 235-256.

2. Arcila, Paola; Mendoza, Yency; Cañón, Óscar y Jaramillo, Jorge. (2010). Comprensión del significado desde Vygotsky, Bruner y Gergen. Diversitas. Perspectivas Psicológicas, 6 (1), pp. 37-49. https://doi.org/10.15332/s1794-9998.2010.0001.03

3. Arcudia, Isabel y Pérez, Francisco. (2014). Historia oral e historia inmediata. La recuperación del sujeto educativo mediante la historiografía crítica. Nóesis. 23 (46), pp. 306-331. https://doi.org/10.20983/noesis.2014.2.11

4. Archila, Mauricio. (2016). El Paro Cívico Nacional del 14 de septiembre de 1977. Un ejercicio de memoria colectiva. Revista de Economía Institucional, 18 (35), pp. 313-318. https://doi.org/10.18601/01245996.v18n35.18

5. Bartolomé, Castor. (2012). El homo sacer y la ciudad antigua. Abandono y gobierno de la vida humana, un diálogo con Giorgio Agamben. En: Bartolomé, Castor. y Duarte, Rubén. (Coord.). La urbe global y el gobierno de la vida humana. Justicia alteridad y memoria en los espacios de poder. (pp. 25-48). Bogotá, D. C.: Universidad Libre de Colombia, Asociación Iberoamericana de Filosofía Política.

6. BBC News Mundo. (2019, noviembre 14). Protestas en Hong Kong: las controvertidas tácticas de los manifestantes en sus enfrentamientos con la policía. https://www.bbc.com/mundo/noticias-internacional-50417321 
7. BBC News Mundo. (2020, enero 31). Qué es el Brexit y otras 5 preguntas básicas para entender la salida del Reino Unido de la Unión Europea. https://www. bbc.com/mundo/noticias-internacional-46521624

8. Bergson, Henri. (2003). Matière et mémoire. Essai sur la relation du corps à I'esprit. Québec: Université du Québec à Chicoutimi. https://doi.org/10.1522/cla.beh. mat

9. Beyer, Harald. (2015). Diagnósticos alternativos sobre la crisis de confianza. Estudios Públicos, 140, pp. 107-136. https://doi.org/10.38178/cep.vi140.179

10. Bonilla, Daniel. (2020). Protesta social y movilizaciones como contextos de interés para la psicología social en Nuestra América. Poiésis, 39, pp. 89-98. https:// doi.org/10.21501/16920945.3756

11. Bonilla, Julián. (2013). Indignados en Europa: lucha contrahegemónica y movilización social. En. Tremolada, Eric. (ed.). Repensando la integración y las integraciones (pp. 491-508). Bogotá, D. C.: Universidad Externado de Colombia.

12. Bustos, Enrique. (1990). El fenómeno de la tortura y su interpretación. En. Martín-Baró, Ignacio (sel.). Psicología social de la guerra. (pp. 211-231). San Salvador: Universidad Católica de El Salvador.

13. Deleuze, Gilles. (1987). El bergsonismo. Madrid: Cátedra.

14. El Comercio. (2020, enero 9). Franceses van a las calles para frenar reforma de las pensiones de Macron. https://www.elcomercio.com/actualidad/francesesmanifestaciones-reforma-pensiones-macron.html

15. Ernst, Ricardo. (2010). Psicología política. En: Villareal, Eduardo y Martínez, Víctor (coords.). (Pre)textos para el análisis político. Disciplinas, reglas y procesos. (pp. 71-91). México, D. F.: Facultad Latinoamericana de Ciencias Sociales.

16. Expansión. (2019, noviembre 15). «La Biblia vuelve a Palacio»: la religión se cuela en la disputa en Bolivia. https://expansion.mx/mundo/2019/11/15/la-bibliavuelve-a-palacio-la-religion-se-cuela-en-la-disputa-en-bolivia

17. Fernández, Pablo. (2003). La psicología política como estética social. Revista Interamericana de Psicología, 37 (2), pp. 253-266.

18. Garzón, Adela. (2008). Teoría y práctica de la psicología política. Información Psicológica, 93, pp. 4-25. Recuperado de: http://bit.ly/2StnleQ

19. Gómez-Suárez, Andrei. (2016). El triunfo del No. La paradoja emocional detrás del plebiscito. Bogotá, D. C.: Ícono.

20. Han, Byung-Chul. (2012). La sociedad del cansancio. Barcelona: Herder.

21. Han, Byung-Chul. (2013). La sociedad de la transparencia. Barcelona: Herder. https://doi.org/10.2307/j.ctvt9k5qb

22. Han, Byung-Chul. (2014a). Psicopolítica. Barcelona: Herder.

23. Han, Byung-Chul. (2014b). En el enjambre. Barcelona: Herder. https://doi. org/10.2307/j.ctvt9k4gh

24. Han, Byung-Chul. (2020). La desaparición de los rituales: una topología del presente. Herder. 
25. Hardt, Michael y Negri, Toni. (2002). Imperio. Barcelona: Paidós.

26. Hardt, Michael y Negri, Toni. (2004). Multitud. Guerra y democracia en la era del imperio. Barcelona: Debate.

27. Infobae. (2019, octubre 10). Violencia en Ecuador: Lenín Moreno denunció que hay guerrilleros de las FARC y enviados de Nicolás Maduro infiltrados en las protestas. https://www.infobae.com/america/america-latina/2019/10/10/violenciaen-ecuador-lenin-moreno-denuncio-que-hay-guerrilleros-de-las-farc-y-enviados-denicolas-maduro-infiltrados-en-las-protestas/

28. Jojoa, Carolina. (2019, noviembre 17). Alertan de posibles riesgos de infiltración durante el paro nacional. El País. https://www.elpais.com.co/colombia/ alertan-de-posibles-riesgos-de-infiltracion-durante-el-paro-nacional.html

29. Lasch, Christopher. (1996). La rebelión de las élites y la traición a la democracia. Barcelona: Oniro.

30. Levitsky, Steven y Ziblatt, Daniel. (2018). Cómo mueren las democracias. Barcelona: Ariel.

31. Marí, Víctor y Santos, Sofía. (2016). Editorial. Commons, 5 (2), pp. 3-7. http:// dx.doi.org/10.25267/COMMONS.2016.v5.i2

32. Martínez-Bascuñán, Máriam. (2015). Democracia y redes sociales: el ejemplo de Twitter. Revista de Estudios Políticos, 168, pp. 175-198. http://dx.doi.org/10.18042/ cepc/rep.168.06

33. Mascato, Rosario. (2009). Tras las huellas de Bergson: fundamentos para

[228] un estudio del intuicionismo en Valle-Inclán. Anales de la Literatura Española Contemporánea, 34 (3), pp. 67-94.

34. Moriconi, Marcelo. (2009). El malestar social y la víctima-cómplice. Polis, 5 (1), pp. 115-142.

35. Ozkan, Mehmet. (2011). El Oriente Medio en la política mundial: un enfoque sistémico. Estudios Políticos, 38, pp. 99-120.

36. Pasquali, Marina. (2019, noviembre 21). El mapa de las protestas en América Latina. Statista. https://es.statista.com/grafico/20043/mapa-de-lasprotestas-en-latinoamerica/

37. Rachman, Gideon. (2019, diciembre 29). 2019, el año en el que las protestas tomaron las calles del mundo. El Cronista. https://www.cronista.com/ financialtimes/Financial-Times-2019-el-ano-en-el-que-las-protestas-tomaron-las-callesdel-mundo-20191229-0016.html

38. Ramos, Rafael. (2019, octubre 19). Un millón de personas se manifiesta en Londres contra la salida de Europa. La Vanguardia. https:/www.lavanguardia.com/ internacional/20191020/471078026199/un-millon-de-personas-se-manifiesta-enlondres-contra-la-salida-de-europa.html

39. Resina, Jorge. (2010). Ciberpolítica, redes sociales y nuevas movilizaciones en España: el impacto digital de los procesos de deliberación y participación ciudadana. Mediaciones Sociales, 7, pp. 143-164. 
40. Sabucedo, José Manual; Rodríguez, Mauro y López, Wilson. (2000). Movilización social contra la violencia política: sus determinantes. Revista Latinoamericana de Psicología, 32 (2), pp. 345-359.

41. Saura, Gemma. (2019, diciembre 1. $\left.{ }^{\circ}\right)$. Los focos de protestas en el mundo. La Vanguardia. https://www.lavanguardia.com/internacional/20191201/471950592846/ focos-protestas-mundo.html

42. Sedeño, Ana. (2015). La visualización de los datos como recurso social: posibilidades educativas y de activismo. Razón y Palabra, 92, pp. 1-14.

43. Sfeir, Paul. (2020, junio 7). De Chile a EEUU - ¿Qué hay detrás de las sospechas de desestabilizadores foráneos? Martí. https://www.radiotelevisionmarti. com/a/de-chile-a-eeuu---qu \% C3\% A9-hay-detr\%C3\%A 1s-de-las-sospechas-dedesestabilizadores-for\%C3\%A1neos-/266207.html

44. Standing, Guy. (2013). El precariado. Una nueva clase social. Barcelona: Pasado y Presente.

45. Torres, Cynthia y Arúe, Raúl. (2013). El mundo de la política y su concepto de acuerdo con militantes universitarios en Tucumán, Argentina. Polis, 9 (1), pp. 109-137.

46. Watson, Peter. (2005). Ideas. Historia intelectual de la humanidad. Barcelona: Crítica. 